\title{
Ontology-based photogrammetric survey in underwater archaeology
}

\author{
Pierre Drap ${ }^{1 *}$, Odile Papini ${ }^{1}$, Jean-Chrisophe Sourisseau ${ }^{2}$ and Timmy Gambin ${ }^{3}$ \\ ${ }^{1}$ Aix-Marseille Université, CNRS, ENSAM, Université De Toulon, LSIS UMR 7296, Domaine \\ Universitaire de Saint-Jérôme, Bâtiment Polytech, 13397, Marseille, France \\ Pierre.Drap@univ-amu.fr, Odile.Papini@univ-amu.fr \\ ${ }^{2}$ Aix Marseille Université, CNRS, Ministère de la Culture et de la Communication, CCJ UMR \\ 7299, 13094, Aix En Provence, France \\ Jean-Christophe. Sourisseauduniv-amu. fr \\ ${ }^{3}$ University of Malta, Msidi, Malte. \\ Timmy. Gambin@um. edu.mt
}

\begin{abstract}
This work addresses the problem of underwater archaeological surveys from the point of view of knowledge. We propose an approach based on underwater photogrammetry guided by a representation of the used knowledge, structured by ontologies. Ontologies and photogrammetry are used together from the survey process to the graphical results. This paper focuses on the use of ontologies during the 3D results exploitation. A JAVA software dedicated to photogrammetry and archaeological survey has been mapped onto an OWL formalism. The use of procedural attachment in a dual representation (JAVA - OWL) of the involved concepts allows to access computational facilities directly from OWL. The use of rules as SWRL illustrates very well this double formalism, also the use of computational capabilities of rules logical expression. We present an application that is able to read the ontology populated with a photogrammetric survey. Once the ontology is read, it is possible to produce a $3 \mathrm{D}$ representation of the individuals and observing graphically the results of logical spatial queries on the ontology. This work is done on a very important archaeological wreck in Malta named Xlendi, probably the most ancient Phoenician wreck of western Mediterranean Sea.
\end{abstract}

Keywords: Underwater Archaeology; Photogrammetry; Ontology; JAVA.

\section{Introduction}

Recent developments in computer vision and photogrammetry, make this latter technique a nearly ideal tool, or at least an essential one, for archaeological survey. In underwater context, it is undeniably a must as there is no efficient alternative.

The main idea of this project is based on the fact that the survey, whether it takes place in the scope of underwater archaeology, relies on a complex well-established corpus, even though it evolves over time. A formalisation of the archaeological knowledge involved, is used to guide the survey.

adfa, p. 1, 2011.

() Springer-Verlag Berlin Heidelberg 2011 
The carried out photogrammetric survey is based on an original approach of underwater photogrammetry that was deployed with the help of a specific instrumental infrastructure provided by COMEX, a partner in the GROPLAN ${ }^{1}$ project [1]. This photogrammetry process, as well as the body of surveyed objects, were formalized in an ontology expressed in OWL2. Our approach is based on procedural attachment; the ontology being seen as a dual of the JAVA class structure that manages the photogrammetric survey and the measurement of artefacts. This allows the establishment of a reasoning for the ontologies as well as the intensive calculations using the JAVA programming language with the same interface. Besides, the ontology used to describe the archaeological artefacts from a measurement point of view is aligned with CIDOC-CRM ontology used for museo-graphical objects [2,3].

The essential of this experimental project is the Xlendi shipwreck, named after the place where it was found off the Gozo coast in Malta. The shipwreck was located by Aurora Trust, an expert in deep-sea detection systems, during a prospecting campaign in 2008. The shipwreck is located near a coastline known for its limestone cliffs that plunge into the sea and whose foundation rests on a continental shelf at an average depth of $100 \mathrm{~m}$ below sea level. The shipwreck itself is therefore exceptional; first due to its configuration and its state of preservation which is particularly well-suited for our experimental 3D modelling project. The examination of the first layer of amphorae also reveals a mixed cargo, consisting of items from Western Phoenicia and Tyrrhenianstyle containers which are both well-matched with the period situated between the end of the VIII and the first half of the VII centuries BC. The historical interest of this wreck, highlighted by our work, which is the first to be performed on this site, creates a real added-value in terms of innovation and the international reputation of the project.

\section{The use of ontologies}

The ontology has been developed to represent the photogrammetry process used for the survey and the process identification, measure, representation of visible archaeological objects. The final ontology is on the one hand an ontology built from a JAVA program modeling the entire photogrammetric process and on the other hand an ontology describing the archaeological artefacts from the point of view of the photogrammetric measure.

The goal is to link the measured artefacts with all the observations used to measure and identify them. One of the main advantage of the photogrammetric process is to provide several 2D representations of the measured artefacts. This first ontology is built from an existing JAVA code in order to represent the concepts used in photogrammetry and to be able to use a reasoner on the ABox representing photogrammetric data. This first mapping from an Object Oriented (OO) formalism to a Description Logic (DL) is relatively easy according to the fact that we have to map a poor semantic formalism toward a richer one [4]. We need to manage both the computational aspects (often heavy

1 http://www.groplan.eu 
in photogrammetry) implanted in the artefacts measurable by photogrammetry and the ontological representation of the same photogrammetric process and surveyed artifacts. The current implementation is based on a double formalism, JAVA, used for computation, photogrammetric algorithms, 3D visualization of photogrammetric data and patrimonial objects, and OWL for the definition of ontologies describing the concepts involved in the measurement process and the link with the measured objects.

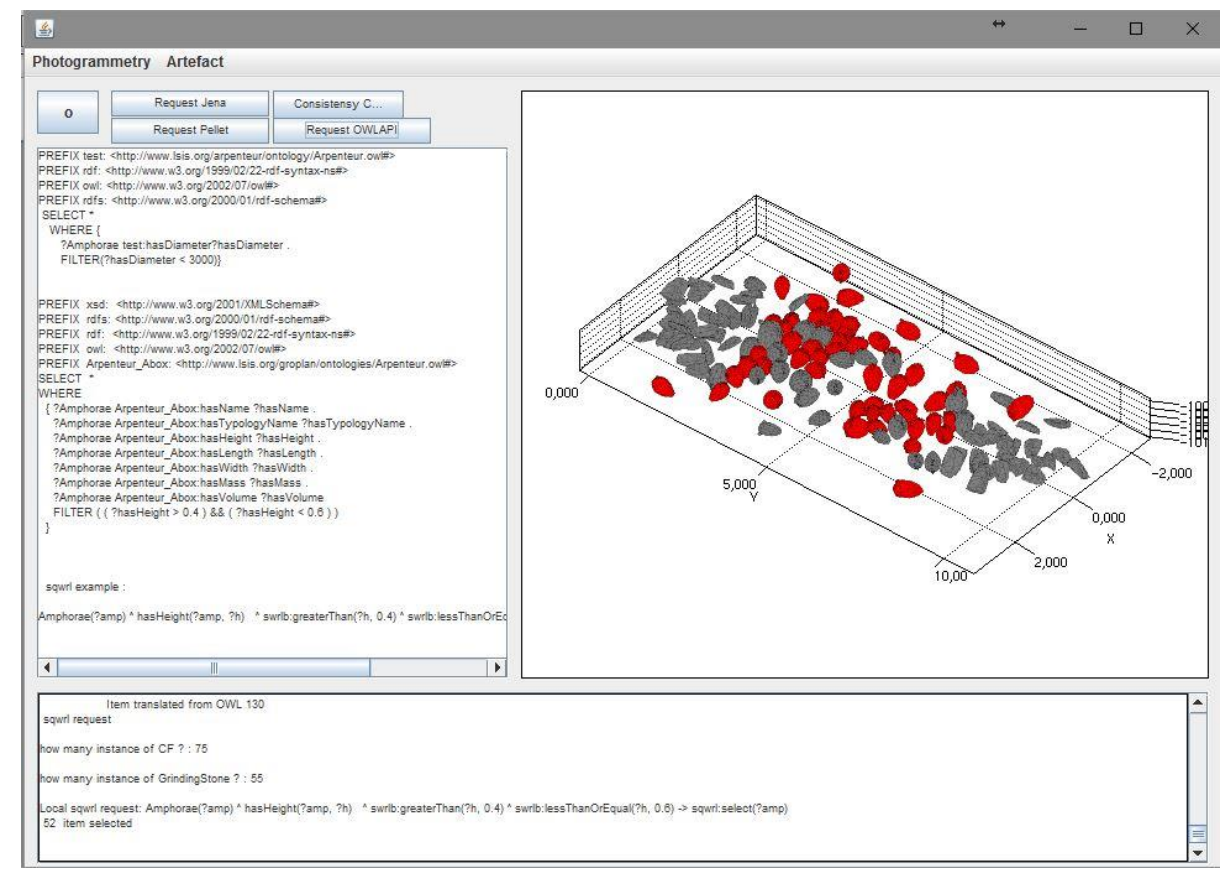

Fig. 1. 3D visualization of a spatial resquest in SWRL:

Amphorae(?a) ^ swrlArp:iscloseTo(?a, "IdTargetAmphora", 6.2) ^ hastypologyName(?a, "Pitecusse_365") -> sqwrl:select(?a)

Means select all amphorae with the typology Pitecusse_365 and at maximum distance of 6.2 meter from the amphorae labelled IdTargetAmphora

The ontology construction in OWL, dual to the JAVA taxonomy, cannot be produced automatically. Each concept of ontology has been constructed in a concern for the representation of fine knowledge from a specific point of view: measurement. Indeed, the same point of view presides over the development of the JAVA taxonomy, but software engineering constraints are superimposed on a point of view strictly linked to knowledge of concepts.

We have abandoned an automatic mapping using JAVA annotation and JAVA beans for a manual extraction even if this is a common way in literature [4-8]. The main advantage of our approach is that it is possible to perform logical queries on both the ontology and the JAVA representation. We can thus read an ontology, visualize in 3D the artefacts present in the ontology as well as the result of SQWRL queries in the 
JAVA viewer. A representation of the artefacts measured on the Xlendi wreck as well as an answer to a SWRL query is shown in Fig. 1.

The approach we have chosen so far, using OWLAPI and the Pellet reasoner, allows for handling SQWRL queries using an extension of SWRL Built-In ${ }^{2}$ packages. SWRL provides a very powerful extension mechanism that allows for implementing user-defined methods in the rules. We have built some spatial operators allowing us to express spatial queries in SWRL, as for example the operator isCloseTo with three arguments which allows for selecting all the amphorae present in a sphere centered on a specific amphora and belonging to a certain typology. ( Fig. 1).

\section{Conclusions and future work}

Based on a procedural attachment approach we built a mechanism which allows for evaluating and visualizing spatial queries from SWRL rules. We are currently extending this approach in a 3D Information System dedicated to archaeological survey based on photogrammetric survey and knowledge representation for spatial reasoning.

\section{Acknowledgement}

This work is partially done in the framework of the projects GROPLAN (ANR-13CORD-0014) and ASPIQ (ANR-12-BS02-0003) funded by ANR, the French agency for scientific research.

\section{References}

1. Drap, P., D. Merad, B. Hijazi, L. Gaoua, M. Nawaf, M. Saccone, B. Chemisky, J. Seinturier, J.-C. Sourisseau, T. Gambin, and F. Castro, Underwater Photogrammetry and Object Modeling: A Case Study of Xlendi Wreck in Malta. Sensors, 2015. 15(12): p. 29802.

2. Gergatsoulis, M., L. Bountouri, P. Gaitanou, and C. Papatheodorou, Mapping cultural metadata schemas to CIDOC conceptual reference model. 2010: Athens. p. 321-326.

3. Niccolucci, F. and A. D'Andrea. An Ontology for 3D Cultural Objects. in The 7th International Symposium on Virtual Reality, Archaeology and Cultural Heritage VAST. 2006.

4. Roy, S. and M.F. Yan, Method and system for creating owl ontology from java, I.T. Limited, Editor. 2012, Google Patents.

5. Kalyanpur, A., D.J.e. Pastor, nez, S. Battle, and J.A. Padget. Automatic Mapping of OWL Ontologies into Java. in SEKE. 2004.

6. Ježek, P. and R. Mouček, Semantic framework for mapping object-oriented model to semantic web languages. Frontiers in Neuroinformatics, 2015. 9(3).

7. Stevenson, G. and S. Dobson, Sapphire: Generating Java Runtime Artefacts from $O W L$ Ontologies, in Advanced Information Systems Engineering Workshops: CAiSE 2011 International Workshops, London, UK, June 20-24, 2011. Proc., Springer. p. 425-436.

8. Horridge, M., H. Knublauch, A. Rector, R. Stevens, and C. Wroe, A Practical Guide To Building OWL Ontologies Using The Protege-OWL Plugin and CO-ODE Tools. 1.0. 2004.

1. https://github.com/protegeproject/swrlapi/wiki/SWRLBuiltInBridge\#SWRL_BuiltIns 